\title{
Microwave spectrum of 1-bromobutane
}

\author{
Jihyun Kim ${ }^{\mathrm{a}}$, Heesu Jang ${ }^{\mathrm{a}}$, Soohyun $\mathrm{Ka}^{\mathrm{a}}$, Daniel A. Obenchain ${ }^{\mathrm{b}}$, Rebecca A. Peebles ${ }^{\mathrm{b}}$, Sean A. \\ Peebles ${ }^{\mathrm{b}}$, and Jung Jin $\mathrm{Oh}^{\mathrm{a}, *}$ \\ ${ }^{\text {a }}$ Department of Chemistry, Research Institute of Global Environment, Sookmyung Women's \\ University, Seoul, 04310, Korea \\ ${ }^{b}$ Department of Chemistry, Eastern Illinois University, 600 Lincoln Ave., Charleston, IL 61920, USA
}

\begin{abstract}
The rotational spectrum of 1-bromobutane has been measured in the range of 8 to $18 \mathrm{GHz}$ using a $480 \mathrm{MHz}$ bandwidth chirped-pulse Fourier transform microwave (CP-FTMW) spectrometer. 1-bromobutane has five conformers; aa, ag, ga, gg, gg'. Spectra for the ${ }^{79} \mathrm{Br}$ isotopic species and ${ }^{81} \mathrm{Br}$ isotopic species were observed and assigned for the three lowest energy conformers, aa, ga, and gg. Consequentially, the rotational constants, nuclear quadrupole coupling constants, and centrifugal distortion constants were determined and the dipole moment of the aa conformer with ${ }^{79} \mathrm{Br}$ was measured. All the experimental data are in good agreement with the $a b$ initio calculations.
\end{abstract}

\section{Keywords}

1-bromobutane

Microwave spectrum

Quadrupole coupling constants

Dipole moment

Ab initio Structure

* Corresponding author.

E-mail address: jjinoh@ sookmyung.ac.kr (J. J. Oh).

\section{Introduction}

The conformational analyses of 1-haloalkanes in gas phase have been performed by Infrared and Raman spectroscopies [1], microwave spectroscopy [2-4], and electron diffraction [5,6]. In the case of 1-bromobutane, Bonham [5] first suggested five possible conformers which are aa, ag, ga, gg and $\mathbf{g g}$ ' in the gas phase and reported their proportions, except for $\mathbf{g g}$ '. Also, four rotational isomers 
(aa, ag, ga, and gg) of 1-halobutane except 1-fluorobutane were observed in the gas, liquid, and glass state by their infrared and Raman bands [1]. Only low resolution microwave spectroscopy (LRMW) [2] was previously applied to observing the rotational spectrum of gaseous 1-bromobutane, and it has confirmed the presence of $\mathbf{a a}, \mathbf{g a}$, and $\mathbf{a g}$ conformers, with some evidence of weak $\mathbf{g g}$ transitions also being present.

As bromine has nuclear spin $I=3 / 2$, which induces hyperfine structure in the molecular spectrum, hyperfine splittings generated by bromine have been observed in the microwave spectra of bromine substituted saturated [7-15], unsaturated [16-22], and ring [23-26] compounds. In this paper, the rotational spectrum and ab initio calculations of 1-bromobutane have been analyzed as one of the series of the 1-bromoalkanes. The structure of 1-bromobutane is also similar with n-butylsilane [27] or n-butylgermane [28], so the same kinds of conformers are expected; aa, ag, ga, gg, and gg' (see Fig. $1)$.

The spectrum in the region of $8 \mathrm{GHz}$ to $18 \mathrm{GHz}$ of this molecule was measured by Chirpedpulse Fourier-transform microwave (CP-FTMW) spectroscopy at Eastern Illinois University (EIU) [2932] and the anti-anti (aa), gauche-anti (ga), and gauche-gauche (gg) conformers were observed. Because of the nuclear spin of bromine, each rotational transition of the molecule shows nuclear quadrupole hyperfine splitting. Complete assignments were performed for the aa, ga, and $\mathbf{g g}$ conformers, and spectroscopic constants including the nuclear quadrupole coupling constants were determined. The electric dipole moments of this molecule were also determined from Stark effect measurements by resonant cavity (Balle-Flygare) Fourier-transform microwave spectroscopy at EIU [33]. The dipole moments of the most intense aa conformer were determined from the measurements using the QSTARKprogram [34] and compared with calculation results.

\section{Experimental}

The rotational spectrum of 1-bromobutane between $8 \mathrm{GHz}$ and $18 \mathrm{GHz}$ was measured by using the $480 \mathrm{MHz}$ bandwidth CP-FTMW spectrometer at EIU [32] with most transitions having full width at half maximum height (FWHM) around $120 \mathrm{kHz}$. The samples were obtained from Aldrich Company. About 1-2 atm of Ar gas was bubbled through a liquid sample of 1-bromobutane just upstream of a pulsed gas valve. A total of 5,000 free induction decays were averaged within each 480 $\mathrm{MHz}$ frequency segment, with these segments later compiled into a full broadband spectrum using a LabView program. 
A resonant cavity (Balle-Flygare) Fourier-transform microwave spectrometer (FTMW) at EIU [33] was used for Stark effect measurements of 1-bromobutane and also for checking the frequency precision of transitions measured on the CP-FTMW spectrometer. The nozzle is perpendicular to the microwave cavity axis and typical line widths of about $20 \mathrm{kHz}$ (FTMW) resulting from Doppler broadening were observed and center frequencies were reproducible to $\pm 2 \mathrm{kHz}$. Two parallel plates supplied DC voltage up to $3 \mathrm{kV}$ for the Stark electric field.

\section{Results and discussion}

\section{Ab initio calculation}

The optimization and energy calculations of 1-bromobutane were carried out at the MP2/6$311+\mathrm{G}(2 \mathrm{~d}, 2 \mathrm{p})$ level using the Gaussian 03 programs [35] to predict relative stability, rotational constants and dipole components of all possible conformers (Fig. 1). In addition, the calculated nuclear quadrupole coupling constants for the main isotopes $\left({ }^{79} \mathrm{Br}, I=3 / 2,50.5 \%\right.$ abundance; ${ }^{81} \mathrm{Br}, I=$ $3 / 2,49.5 \%$ ) were also obtained. The results are listed in Table 1.

In our calculation results, the gg conformer of 1-bromobutane has the lowest energy. O. Takahashi [36] and coworkers, however, reported that the aa conformer has the lowest Gibbs energy in the results of calculation at the different basis set, MP2/6-311G(d,p). This is consistent with the earlier electron diffraction study on the structure of 1-bromobutane in the gas phase using sector-microphotometer performed by Bonham [5] which suggested that the conformer percentage of aa form is $36 \%$ and $\mathbf{g g}$ form is $16 \%$ at $25^{\circ} \mathrm{C}$. Another electron diffraction experiment [6] at $18^{\circ} \mathrm{C}$ reported that the percentages are reversed; gg conformer is $38 \pm 34 \%$, but aa conformer is $21 \pm 14 \%$.

As shown in Table1, the calculated energy differences between $\mathbf{a a}$, ga, and gg conformers are very small $(\sim 0.1 \mathrm{~kJ} / \mathrm{mol})$, so it is reasonable that the stability order can vary with different basis set, level of calculation and depending on whether Gibbs energies or internal energies are compared. The zero point energy (ZPE) was not included to compare the relative stability since-the energy differences are relatively small and therefore the relative stabilities are uncertain with or without ZPE corrections. Experimentally, the present study utilized a supersonic expansion, so populations are not directly comparable with room temperature ab initio calculations. Though the most stable molecule was not obvious, quite a few lines of three conformers (aa, ga, and $\mathbf{g g}$ ) in the spectrum are expected to be strong because those conformers were detected in the previous electron diffraction study [5]. Heavy atom structural parameters from the $a b$ initio calculations are listed in Table 2. 


\section{Microwave spectra}

The $8 \mathrm{GHz}$ to $18 \mathrm{GHz}$ spectrum was measured using CP-FTMW and resonant cavity FTMW at Eastern Illinois University (EIU) [32,33]. Rotational constants, centrifugal distortion constants and nuclear quadrupole coupling constants were determined and fitted by using Watson's $\mathrm{S}$ reduced Hamiltonian in its $I^{r}$ representation [37] using the SPFIT program [38].

All conformers are determined to be prolate asymmetric type rotors ( $\kappa \sim-0.87$ to -0.99 for all five conformers). Due to bromine nuclear quadrupole coupling, rotational transitions exhibited hyperfine splitting [21-23]. The nuclear spin $\boldsymbol{I}$ is coupled with the molecular rotational angular momentum $\boldsymbol{J}$ to form a resultant $\boldsymbol{F}$. The total angular momentum of the molecule with nuclear coupling is thus represented by $\boldsymbol{F}$ rather than $\boldsymbol{J}$, which designates the total angular momentum exclusive of nuclear spin. The good quantum numbers are thus $\boldsymbol{F}, \boldsymbol{M}_{\boldsymbol{F}}, \boldsymbol{J}$ and $\boldsymbol{I} . \quad \boldsymbol{F}$ is the quantum number for total angular momentum including nuclear spin, and $\boldsymbol{M}_{\boldsymbol{F}}$ is the projection of total angular momentum including nuclear spin on space-fixed axis. The new angular momentum quantum numbers are as follows.

$$
\begin{gathered}
F=J+I, J+I-1, J+I-2, \ldots,|J-I| \\
M_{F}=F, F-1, F-2, \ldots,-F
\end{gathered}
$$

Selection rules for hyperfine transitions in rotational absorption spectra are

$$
J \rightarrow J+1, F \rightarrow F, F \rightarrow F \pm 1, I \rightarrow I .
$$

In brief, when the $J>I$, there are $2 I+1$ components for $F \rightarrow F+1,2 I$ components for $F \rightarrow F$, and $2 I-1$ components for $F \rightarrow F-1$. The $F \rightarrow F+1$ components are strong, while the $F \rightarrow F-1$ components become weaker and are thus generally undetectable.

In the case of 1-bromobutane, the split lines consisted of only four strong $F \rightarrow F+1$ transition components (see Fig. 2). The ${ }^{13} \mathrm{C}$ isotopomer spectra could not be found in the spectrum without enrichment.

For aa conformer, the series of the strongest $a$-type transitions with $J$ from 5 to 10 were found and assigned by their relative intensity and characteristic hyperfine splitting. A total of 86 and 98 hyperfine components each for ${ }^{79} \mathrm{Br}$ and ${ }^{81} \mathrm{Br}$ were assigned. The aa conformer assumes $\mathrm{C}_{\mathrm{s}}$ symmetry, so there are only three independent nuclear quadrupole coupling tensor components, including an offdiagonal component $\chi_{a b}$ (see Table 3). Fitted quadrupole coupling tensor components all agree well with the ab initio calculations shown in Table 1 . For example, experimental $\chi_{\text {aa }}$ is $363 \mathrm{MHz}$, and it is very 
consistent with the calculated value, $361 \mathrm{MHz}$. Only two distortion constants ( $\mathrm{D}_{\mathrm{J}}, \mathrm{D}_{\mathrm{JK}}$ ) are determined for the 1-bromobutane aa conformer, and other conformers have three distortion constants including $\mathrm{d}_{1}$. All the observed transitions are listed in Table 4.

The ga conformer, unlike the aa conformer, has relatively large $\mu_{b}$ dipole component $\left(\mu_{a}=\right.$ $1.621 \mathrm{D}, \mu_{b}=1.257 \mathrm{D}$ ); this allows predictions of $b$-type transitions with intensities comparable to the $a$-type transitions. As a result, the series of strong $b$-type transitions with $J$ from 3 to 8 were found and assigned (See Table 5 and 6).

For the gg conformer, only $a$-type transitions with $J$ from 3 to 6 were measured and assigned. A search for $b$-type transitions, which were expected to appear at less than $10 \%$ the intensity of $a$-type transitions, was not successful because of other nearby transitions and low intensity. Interestingly, the spectrum for the gg conformer (Table 7) was noticeably less intense than that for the aa and ga forms. The larger $\mu_{a}$ for the aa conformer leads to stronger $a$-type transitions for that conformer, but that does not explain the ga conformer spectrum being more intense than gg since $\mu_{a}$ for $\mathbf{g g}$ is slightly larger than that for ga (See Table 1). Although making quantitative comparisons of relative abundances is challenging using the $480 \mathrm{MHz}$ spectrometer due to instrumental effects on intensities during the construction of the full broadband spectrum, when we take into account the predicted dipole moment components for these three species, we must conclude that the gg conformer seems to be less abundant than our calculations predict, in agreement with the previous LRMW study [2] and one of the electron diffraction results [5].

The nuclear electric quadrupole coupling tensors $\left(\chi_{x x}, \chi_{y y}\right.$, and $\left.\chi_{z z}\right)$ in the principal axes of bromine were derived by diagonalization of experimentally determined three or five nuclear quadrupole coupling tensor components (Table 3). The $\chi_{z z}(\mathrm{MHz})$ for ${ }^{79} \mathrm{Br}$ are 543.4, 539.7 and 536.2 to the aa, ga, and gg conformers, while for ${ }^{81} \mathrm{Br}$, it ranges from 448 to $454 \mathrm{MHz}$ [34].

Those quadrupole coupling tensors for ${ }^{79} \mathrm{Br}$ and ${ }^{81} \mathrm{Br}$ were compared respectively with the values of methyl bromide $\left(\mathrm{CH}_{3} \mathrm{Br}\right)[15,16]$, ethyl bromide $\left(\mathrm{CH}_{3} \mathrm{CH}_{2} \mathrm{Br}\right)[12,13]$ and n-propyl bromide $\left(\mathrm{CH}_{3} \mathrm{CH}_{2} \mathrm{CH}_{2} \mathrm{Br}\right)$ [14]. They are presented in Table 8. In the case of methyl bromide, only $\chi_{z z}$ were observed and $\chi_{\mathrm{xx}}$ and $\chi_{\mathrm{yy}}$ are determined by symmetry. The quadrupole coupling tensors of 1bromobutane are more similar to those of ethyl bromide rather than n-propyl bromide. Among three conformers, the aa conformer has the closest values to the tensors of ethyl bromide studied by Inagusa and Hayashi [12], while the other two conformers are similar to the tensors of ethyl bromide investigated by Flanagan and Pierce [13].

\section{Dipole moment}


The Stark effect shifts and splits spectral lines of atoms and molecules when the system is subjected to an electric field. It allows measurement of the electric dipole moment of gaseous molecules. The measurement for the aa conformer of 1-bromobutane with ${ }^{79} \mathrm{Br}$ was performed on Stark components of the $5_{1,5}-4_{1,4} F=9 / 2 \leftarrow 7 / 2$ rotational transition at $8922.5465 \mathrm{MHz}$, the $5_{1,4}-4_{1,3} F=9 / 2$ $\leftarrow 7 / 2$ transition at $9075.5248 \mathrm{MHz}$, and the $6_{1,6}-5_{1,5} F=9 / 2 \leftarrow 7 / 2$ transition at $10700.8613 \mathrm{MHz}$. A total of 11 components were measured with $\Delta M_{F}=0$ selection rules. The Stark shifts have been measured for applied fields up to $140 \mathrm{~V} / \mathrm{cm}$. Some Stark components weren't measurable because the sublevel lines usually lose intensity quickly as more electric field is applied. The $5_{1,4}-4_{1,3} F=9 / 2 \leftarrow$ $7 / 2$ transition has four components, but only three components were confirmed: $M_{F}=1 / 2,3 / 2$, and 5/2 (except for $7 / 2)$. The aa conformer of 1-bromobutane has a symmetry plane ( $\mathrm{C}_{\mathrm{s}}$ symmetry). Thus, it is expected that the aa conformer would possess non-zero $\mu_{a}$ and $\mu_{b}$ dipole moment components, and two components of the dipole moment were determined. The resulting experimental dipole moments are listed in Table 9 with $a b$ initio calculated dipole components. The experimental dipole moments are very consistent with the calculated dipole moments. The dipole moments are influenced by basis set of calculation, so further calculation applying various basis sets might predict more exact dipole moments. All the measured (points) and the calculated (continuous lines) frequency shifts of $\Delta M_{F}=$ 0 components are plotted in Fig. 3.

\section{Conclusions}

The 8-18 GHz microwave spectrum of 1-bromobutane was measured using CP-FTMW, and further studies were performed using resonant cavity FTMW. The spectroscopic constants were determined by fit and compared with the $a b$ initio calculated results. The rotational constants for the three stable conformers (aa, ga, and gg) of 1-bromobutane have been accurately determined. The centrifugal distortion constants and bromine nuclear quadrupole coupling constants were also obtained. The absence of an ag conformer is somewhat puzzling, particularly in light of its observation in a previous low resolution microwave study [2]. We assume that our failure to detect it in the present work is due to a small population, and/or relaxation to other conformers in the supersonic expansion since it is predicted to lie $2.5 \mathrm{~kJ} / \mathrm{mol}$ above the other three conformers (See Table 1) [39].

The calculated rotational constants and nuclear quadrupole coupling constants are all in good agreement with the experimental results. The close agreement between spectroscopic parameters with the $a b$ initio calculation at the MP2/6-311+G(2d,2p) level shows good credibility of the theoretical calculation at this level for 1-bromobutane. 
Based on Stark effect, dipole moment components for aa conformer were determined and compared with $a b$ initio values optimized at the level of MP2/6-311+G(2d,2p). A good agreement between experimental and calculated dipole moments suggests that the structural information of aa conformer also would fit with the results of calculation.

The rotational spectra indicate that the gg conformer is less abundant than our calculations suggested and so exact abundances of the other conformers are still unclear. Higher level calculations (including ZPE corrections, which were neglected in the current work) might lead to insight on barriers between conformers, whether conformer relaxation is significant in the supersonic expansion and how sensitive the stability order is to computational method and basis set effects. Our results appear to be consistent with electron diffraction and low resolution microwave studies performed on room temperature gas phase samples; thus, it is also unclear what effect the supersonic expansion has on relative populations of the conformers in this case. 


\section{Acknowledgements}

This work was supported by the National Research Foundation of Korea (NRF) grant funded by the Korea government (MSIP) (No. NRF-2013R1A2A1A03070600). Construction of the CP-FTMW spectrometer at Eastern Illinois University was supported by National Science Foundation Research at Undergraduate Institutions (NSF-RUI) grant \#0809387.

\section{References}

[1] Y. Ogawa, S. Imazeki, H. Yamaguchi, H. Matsuura, I. Harada, T. Shimanouchi, Bull. Chem. Soc. Jpn. 51 (1978) 748-767.

[2] W.E. Steinmetz, F. Hickernell, I.K. Mun, L.H. Scharpen, J. Mol. Spectrosc. 68 (1977) 173182.

[3] S. Melandri, P.G. Favero, W. Caminati, Laura B. Favero, A. Degli Esposti, J. Chem. Soc. Faraday Trans. 93 (1997) 2131-2137.

[4] L.B. Favero, A. Maris, A.D. Esposti, P.G. Favero, W. Caminati, G. Pawelke, Chem. - A Eur. J. 6 (2000) 3018-3025.

[5] F.A. Momany, R.A. Bonham, W.H. McCoy, J. Am. Chem. Soc. 85 (1963) 3077-3080.

[6] K. Aarset, K. Hagen, R. Stølevik, S. Per Christian, Struct. Chem. 6 (1995) 197-205.

[7] R.H. Schwendeman, F.L. Tobiason, J. Chem. Phys. 43 (1965) 201.

[8] A.C. Ferguson, J. Chem. Phys. 53 (1970) 1851.

[9] C. Ikeda, T. Inagusa, M. Hayashi, J. Mol. Spectrosc. 135 (1989) 334-348.

[10] M. Meyer, W. Stahl, H. Dreizler, J. Mol. Spectrosc. 151 (1992) 243-259.

[11] Y. Tatamitani, S. Kuwano, K. Fuchigami, S. Oe, T. Ogata, J. Mol. Spectrosc. 196 (1999) 189 196.

[12] T. Inagusa, M. Hayashi, J. Mol. Spectrosc. 129 (1988) 160-171.

[13] C. Flanagan, L. Pierce, J. Chem. Phys. 38 (1963) 2963-2969.

[14] Y. Niide, I. Ohkoshi, M. Takano, J. Mol. Spectrosc. 89 (1981) 387-396.

[15] S. Carocci, P. Minguzzi, M. Tonelli, A. Dilieto, J. Mol. Spectrosc. 160 (1993) 359-370.

[16] J.D. Langridge, B.E. Long, S.A. Cooke, J. Mol. Struct. 1000 (2011) 24-28.

[17] Y. Niide, M. Takano, T. Satoh, Y. Sasada, J. Mol. Spectrosc. 63 (1976) 108-119.

[18] B.P. van Eijck, V.M. Stolwijk, J. Mol. Spectrosc. 111 (1985) 164-172.

[19] Y.J. Xu, W. Jager, M.C.L. Gerry, I. Merke, J. Mol. Spectrosc. 160 (1993) 258-271.

[20] I. Merke, N. Vaeck, D. Petitprez, G. Wlodarczak, J. Mol. Struct. 780-781 (2006) 306-309.

[21] J. Shimada, Y. Tatamitani, B. Liu, T. Ogata, J. Mol. Struct. 612 (2002) 155-166. 
[22] R.A. Powoski, G.S. Grubbs, S.A. Cooke, J. Mol. Struct. 963 (2010) 106-110.

[23] H. Li, M.C.L. Gerry, W. Lewis-Bevan, J. Mol. Spectrosc. 144 (1990) 51-69.

[24] E. Rosenthal, B.P. Dailey, J. Chem. Phys. 43 (1965) 2093.

[25] W. Caminati, A.M. Mirri, Chem. Phys. Lett. 12 (1971) 127-130.

[26] W. Caminati, P. Forti, Chem. Phys. Lett. 15 (1972) 343-349.

[27] H.D. Stidham, A.J. LaPlante, J.-J. Oh, D.A. Obenchain, S.A. Peebles, R.A. Peebles, C.J. Wurrey, E. Marrow, G.A. Guirgis, J. Mol. Struct. 1003 (2011) 31-40.

[28] A.J. LaPlante, H.D. Stidham, S.A. Peebles, R.A. Peebles, C.J. Wurrey, G.A. Guirgis, J. Mol. Struct. 985 (2011) 5-13.

[29] G.G. Brown, B.C. Dian, K.O. Douglass, S.M. Geyer, B.H. Pate, J. Mol. Spectrosc. 238 (2006) 200-212.

[30] G.G. Brown, B.C. Dian, K.O. Douglass, S.M. Geyer, S.T. Shipman, B.H. Pate, Rev. Sci. Instrum. 79 (2008) 053103.

[31] G.S. Grubbs, C.T. Dewberry, K.C. Etchison, K.E. Kerr, S.A. Cooke, Rev. Sci. Instrum. 78 (2007) 096106.

[32] D.A. Obenchain, A.A. Elliott, A.L. Steber, R.A. Peebles, S.A. Peebles, C.J. Wurrey, G.A. Guirgis, J. Mol. Spectrosc. 261 (2010) 35-40.

[33] T.J. Balle, Rev. Sci. Instrum. 52 (1981) 33.

[34] Z. Kisiel, J. Kosarzewski, B.A. Pietrewicz, L. Pszczółkowski, Chem. Phys. Lett. 325 (2000) $523-530$.

[35] Gaussian 03, Revision D.01, M. J. Frisch, G. W. Trucks, H. B. Schlegel, G. E. Scuseria, M. A. Robb, J. R. Cheeseman, J. A. Montgomery, Jr., T. Vreven, K. N. Kudin, J. C. Burant, J. M. Millam, S. S. Iyengar, J. Tomasi, V. Barone, B. Mennucci, M. Cossi, G. Scalmani, N. Rega, G. A. Petersson, H. Nakatsuji, M. Hada, M. Ehara, K. Toyota, R. Fukuda, J. Hasegawa, M. Ishida, T. Nakajima, Y. Honda, O. Kitao, H. Nakai, M. Klene, X. Li, J. E. Knox, H. P. Hratchian, J. B. Cross, V. Bakken, C. Adamo, J. Jaramillo, R. Gomperts, R. E. Stratmann, O. Yazyev, A. J. Austin, R. Cammi, C. Pomelli, J. W. Ochterski, P. Y. Ayala, K. Morokuma, G. A. Voth, P. Salvador, J. J. Dannenberg, V. G. Zakrzewski, S. Dapprich, A. D. Daniels, M. C. Strain, O. Farkas, D. K. Malick, A. D. Rabuck, K. Raghavachari, J. B. Foresman, J. V. Ortiz, Q. Cui, A. G. Baboul, S. Clifford, J. Cioslowski, B. B. Stefanov, G. Liu, A. Liashenko, P. Piskorz, I. Komaromi, R. L. Martin, D. J. Fox, T. Keith, M. A. Al-Laham, C. Y. Peng, A. Nanayakkara, M. Challacombe, P. M. W. Gill, B. Johnson, W. Chen, M. W. Wong, C. Gonzalez, and J. A. Pople, Gaussian, Inc., Wallingford CT, 2004.

[36] O. Takahashi, K. Yamasaki, Y. Kohno, K. Ueda, H. Suezawa, M. Nishio, Chem. Phys. Lett. 440 (2007) 64-69.

[37] J.K.G. Watson, Vib. Spectra. Struct. 6 (1977) 1-89. 
[38] H.M. Pickett, J. Mol. Spectrosc. 148 (1991) 371-377.

[39] R. S. Ruoff, T. D. Klots, T. Emilsson, and H. S. Gutowsky, J. Chem. Phys. 93(5), (1990) 31423150. 

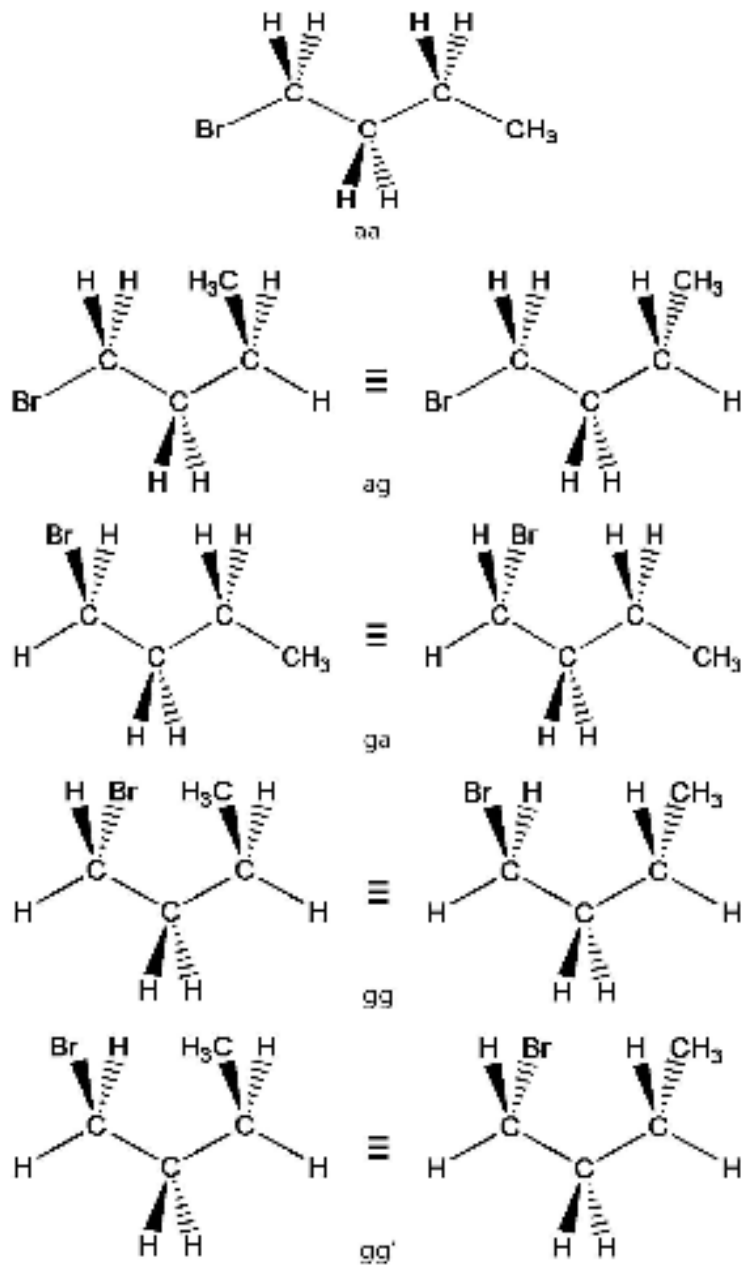

Fig. 1. Structure of the five conformers for 1-bromobutane. 


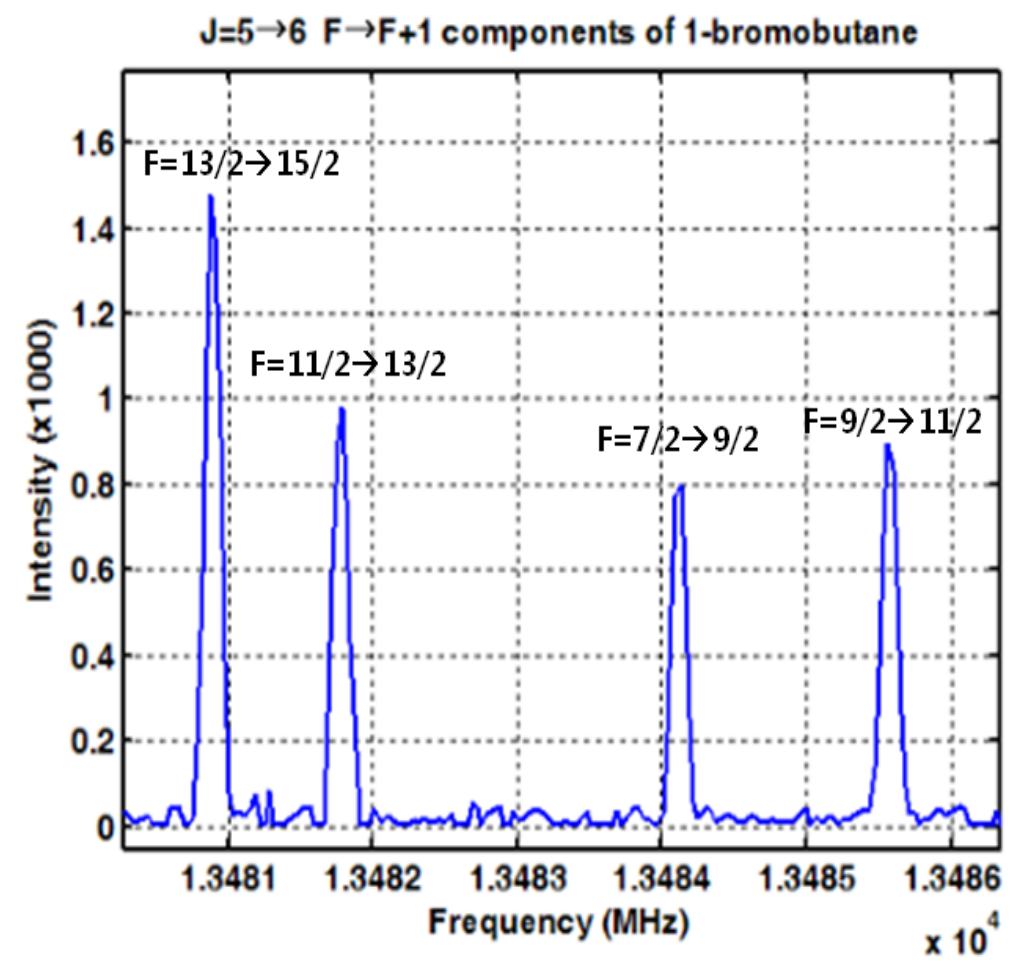

Fig. 2. Observed $\mathrm{J}=5_{15} \rightarrow 6_{16} ; \mathrm{F} \rightarrow \mathrm{F}+1$ rotational transitions of the ga conformer of ${ }^{79} \mathrm{Br} 1$ bromobutane. 

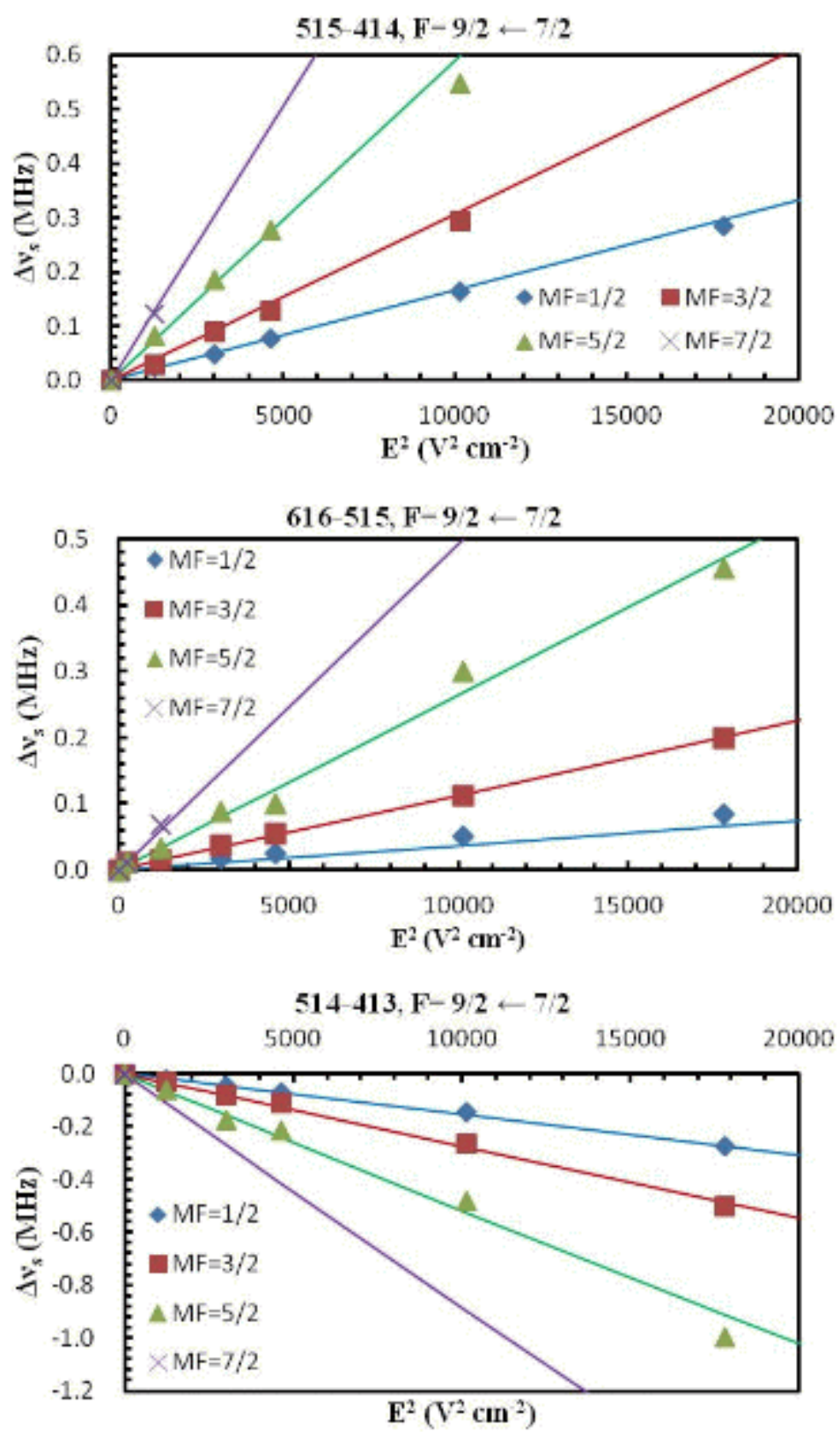

Fig. 3. Observed (point) and calculated (continuous lines) frequency shifts for some of the observed $\Delta \mathrm{M}_{\mathrm{F}}=0$ Stark components in $\mathrm{CH}_{3} \mathrm{CH}_{2} \mathrm{CH}_{2} \mathrm{CH}_{2}{ }^{79} \mathrm{Br}$ aa conformer. 
Table 1

$A b$ initio calculated energy ${ }^{\mathrm{a}}$, dipole moments and spectroscopic constants for the five conformers of 1-bromobutane.

\begin{tabular}{|c|c|c|c|c|c|c|}
\hline & \multirow{2}{*}{$\mathrm{E}$ (hartree) } & \multirow{2}{*}{$\Delta \mathrm{E}(\mathrm{kJ} / \mathrm{mol})$} & \multirow{2}{*}{ Stability } & \multicolumn{3}{|c|}{ Relative Abundances } \\
\hline & & & & $\mathrm{T}=5 \mathrm{~K}$ & $\mathrm{~T}=10 \mathrm{~K}$ & $\mathrm{~T}=298 \mathrm{~K}$ \\
\hline gg & -2729.91262 & 0.000 & 1 & 1.0000 & 1.0000 & 1.0000 \\
\hline aa & -2729.91259 & 0.079 & 2 & 0.1483 & 0.3852 & 0.9685 \\
\hline ga & -2729.91258 & 0.096 & 3 & 0.0984 & 0.3137 & 0.9618 \\
\hline ag & -2729.91164 & 2.561 & 4 & 0.0000 & 0.0000 & 0.3555 \\
\hline \multirow[t]{2}{*}{ gg' $^{\prime}$} & -2729.90858 & 10.599 & 5 & 0.0000 & 0.0000 & 0.0138 \\
\hline & \multicolumn{2}{|c|}{ gg } & $\mathbf{a a}^{\mathbf{b}}$ & ga & ag & $\mathbf{g g}^{\prime}$ \\
\hline $\mathrm{A}(\mathrm{MHz})$ & \multicolumn{2}{|c|}{6126.63794} & 16054.11243 & 8271.33080 & 12122.51662 & 5723.00672 \\
\hline $\mathrm{B}(\mathrm{MHz})$ & \multicolumn{2}{|c|}{1508.25371} & 915.13169 & 1210.30632 & 1019.85790 & 1629.57403 \\
\hline $\mathrm{C}(\mathrm{MHz})$ & \multicolumn{2}{|c|}{1385.35718} & 884.61391 & 1111.00077 & 984.44351 & 1349.47750 \\
\hline$\chi_{\mathrm{aa}}(\mathrm{MHz})$ & \multicolumn{2}{|c|}{151.57616} & 361.14646 & 89.08847 & 463.04856 & 116.40059 \\
\hline$\chi_{\mathrm{bb}}-\chi_{\mathrm{ccc}}(\mathrm{MHz})$ & \multicolumn{2}{|c|}{156.39767} & 174.66810 & 398.25101 & -35.35525 & 318.83450 \\
\hline$\chi_{\mathrm{ab}}(\mathrm{MHz})$ & \multicolumn{2}{|c|}{-328.51140} & -325.45810 & 386.29567 & 103.65999 & 381.13331 \\
\hline$\left.\chi_{\mathrm{ac}} \mathrm{MHz}\right)$ & \multicolumn{2}{|c|}{217.66350} & & -70.97172 & -198.55955 & 129.55247 \\
\hline$\chi_{\mathrm{bc}}(\mathrm{MHz})$ & \multicolumn{2}{|c|}{-170.37734} & & -85.52451 & -30.64685 & 130.34058 \\
\hline$\kappa$ (Kappa) & \multicolumn{2}{|c|}{-0.948} & -0.996 & -0.972 & -0.994 & -0.872 \\
\hline$\mu_{\mathrm{a}}(\mathrm{D})$ & \multicolumn{2}{|c|}{1.705} & 2.179 & 1.621 & 2.278 & 1.671 \\
\hline$\mu_{\mathrm{b}}(\mathrm{D})$ & \multicolumn{2}{|c|}{1.041} & 0.744 & 1.257 & 0.041 & 1.246 \\
\hline$\mu_{\mathrm{c}}(\mathrm{D})$ & \multicolumn{2}{|c|}{0.478} & 0.000 & 0.216 & 0.391 & 0.291 \\
\hline$\mu_{\mathrm{tot}}(\mathrm{D})$ & \multicolumn{2}{|c|}{2.054} & 2.303 & 2.063 & 2.312 & 2.104 \\
\hline
\end{tabular}

${ }^{\mathrm{b}}$ The aa conformer has $\mathrm{C}_{\mathrm{s}}$ symmetry, so there are only three nuclear quadrupole coupling tensor components. 
Table 2

Heavy atom structural parameters of 1-bromobutane from $a b$ initio optimization at MP2/6-311+G(2d,2p) level. (See Fig.1 for conformer structure)

\begin{tabular}{|c|c|c|c|c|c|}
\hline & aa & ag & ga & gg & $g^{\prime}$ \\
\hline $\mathrm{R}\left(\mathrm{Br}-\mathrm{C}_{\mathrm{l}}\right)(\AA)$ & 1.953 & 1.954 & 1.957 & 1.957 & 1.959 \\
\hline $\mathrm{R}\left(\mathrm{C}_{1}-\mathrm{C}_{2}\right)(\AA)$ & 1.513 & 1.514 & 1.514 & 1.515 & 1.520 \\
\hline $\mathrm{R}\left(\mathrm{C}_{2}-\mathrm{C}_{3}\right)(\AA)$ & 1.528 & 1.531 & 1.524 & 1.527 & 1.529 \\
\hline $\mathrm{R}\left(\mathrm{C}_{3}-\mathrm{C}_{4}\right)(\AA)$ & 1.526 & 1.527 & 1.525 & 1.526 & 1.527 \\
\hline$\angle\left(\mathrm{Br}-\mathrm{C}_{1}-\mathrm{C}_{2}\right)\left(^{\circ}\right)$ & 111.93 & 111.57 & 112.24 & 112.26 & 113.98 \\
\hline$\angle\left(\mathrm{C}_{1}-\mathrm{C}_{2}-\mathrm{C}_{3}\right)\left(^{\circ}\right)$ & 111.10 & 111.89 & 114.19 & 114.92 & 115.51 \\
\hline$\angle\left(\mathrm{C}_{2}-\mathrm{C}_{3}-\mathrm{C}_{4}\right)\left(^{\circ}\right)$ & 111.97 & 113.61 & 111.97 & 113.33 & 115.52 \\
\hline$\tau\left(\mathrm{Br}-\mathrm{C}_{1}-\mathrm{C}_{2}-\mathrm{C}_{3}\right)\left(^{\circ}\right)$ & 180.00 & -177.67 & -66.11 & -60.39 & -84.82 \\
\hline$\tau\left(\mathrm{C}_{1}-\mathrm{C}_{2}-\mathrm{C}_{3}-\mathrm{C}_{4}\right)\left({ }^{\circ}\right)$ & 180.00 & -64.77 & -177.63 & -55.63 & 65.80 \\
\hline
\end{tabular}


Table 3

Spectroscopic constants for aa, ga, and gg conformers of 1-bromobutane.

\begin{tabular}{|c|c|c|c|c|c|c|}
\hline & \multicolumn{2}{|c|}{ aa } & \multicolumn{2}{|c|}{ ga } & \multicolumn{2}{|c|}{ gg } \\
\hline & ${ }^{79} \mathrm{Br}$ & ${ }^{81} \mathrm{Br}$ & ${ }^{79} \mathrm{Br}$ & ${ }^{81} \mathrm{Br}$ & ${ }^{79} \mathrm{Br}$ & ${ }^{81} \mathrm{Br}$ \\
\hline $\mathrm{A}(\mathrm{MHz}, \mathrm{calc})^{\mathrm{a}}$ & 16054.1124 & & 8271.3308 & & 6126.6379 & \\
\hline $\mathrm{B}(\mathrm{MHz}, \text { calc })^{\mathrm{a}}$ & 915.1317 & & 1210.3063 & & 1508.2537 & \\
\hline $\mathrm{C}(\mathrm{MHz}, \text { calc })^{\mathrm{a}}$ & 884.6139 & & 1111.0008 & & 1385.3572 & \\
\hline $\mathrm{A}(\mathrm{MHz})$ & 15770.66( 99) & $15760.27(131)$ & $8196.3730(66)$ & $8183.7297(61)$ & $6240.618(278)$ & $6235.48(81)$ \\
\hline $\mathrm{B}(\mathrm{MHz})$ & $914.89072(63)$ & $907.47581(61)$ & $1207.26281(105)$ & $1197.95218(96)$ & $1463.8266(41)$ & $1452.1707(44)$ \\
\hline $\mathrm{C}(\mathrm{MHz})$ & $883.90660(67)$ & $876.95290(62)$ & $1107.67707(78)$ & $1099.61264(77)$ & $1352.8742(36)$ & $1342.6930(41)$ \\
\hline $\mathrm{D}_{\mathrm{J}}(\mathrm{kHz})$ & $0.0634(32)$ & $0.05829(301)$ & $0.3171(77)$ & $0.3116(64)$ & $1.5084(278)$ & $1.4786(282)$ \\
\hline $\mathrm{D}_{\mathrm{JK}}(\mathrm{kHz})$ & $-3.091(115)$ & $-3.004(85)$ & $-5.639(111)$ & $-5.675(97)$ & $-12.775(238)$ & $-13.00(101)$ \\
\hline $\mathrm{d}_{1}(\mathrm{kHz})$ & & & $-0.0511(49)$ & $-0.0442(49)$ & $-0.278(38)$ & $-0.281(32)$ \\
\hline$\chi_{\mathrm{aa}}(\mathrm{MHz})$ & $363.04(34)$ & $303.67(33)$ & $87.17(10)$ & 73.83(93) & $157.83(11)$ & $132.69(22)$ \\
\hline$\chi_{\mathrm{bb}}-\chi_{\mathrm{ccc}}(\mathrm{MHz})$ & $184.90(113)$ & $153.21(110)$ & $420.24(6)$ & $350.20(6)$ & $141.98(55)$ & $117.95(74)$ \\
\hline$\chi_{\mathrm{ab}}(\mathrm{MHz})$ & $-337.81(13)$ & $-282.01(20)$ & $394.70(44)$ & $329.92(43)$ & $-336.11(28)$ & $-280.1(36)$ \\
\hline$\chi_{\mathrm{ac}}(\mathrm{MHz})$ & & & $-72.26(236)$ & $-59.81(271)$ & $221.62(31)$ & $186.5(64)$ \\
\hline$\chi_{\mathrm{bc}}(\mathrm{MHz})$ & & & $-87.38(18)$ & $-74.04(149)$ & $-172.68(76)$ & $-144.24(68)$ \\
\hline$\chi_{x x}$ & $-269.48(48)$ & $-225.51(48)$ & $-264.89(197)$ & $-220.16(242)$ & $-264.88(54)$ & $-221.98(182)$ \\
\hline$\chi_{y y}$ & $-273.97(59)$ & $-228.44(58)$ & $-274.83(154)$ & $-230.95(210)$ & $-271.29(39)$ & $-226.31(393)$ \\
\hline$\chi_{z z}$ & $543.45(32)$ & $453.95(33)$ & $539.71(63)$ & $451.11(84)$ & $536.17(46)$ & $448.29(465)$ \\
\hline $\mathrm{N}^{\mathrm{b}}$ & $86(23)$ & $98(26)$ & $99(26)$ & $105(28)$ & $47(14)$ & $35(9)$ \\
\hline$\Delta v_{\mathrm{rms}}(\mathrm{kHz})^{\mathrm{c}}$ & 6.08 & 5.10 & 3.58 & 5.12 & 3.90 & 5.71 \\
\hline$\kappa($ Карра) & -0.996 & -0.996 & -0.972 & -0.972 & -0.955 & -0.955 \\
\hline
\end{tabular}

${ }^{\mathrm{a}}$ Calculation was performed using MP2/6-311+G(2d,2p) Gaussian 03 program.

${ }^{\mathrm{b}} \mathrm{N}$ is the number of fitted hyperfine components; the number in parentheses shows the number of separate rotational transitions.

${ }^{\mathrm{c}}$ Standard deviation of the fit in $\mathrm{kHz}$. 
Table 4

Observed $a$-type transition frequencies for aa conformer of 1-bromobutane.

\begin{tabular}{|c|c|c|c|c|c|c|c|c|c|}
\hline \multirow{2}{*}{$\begin{array}{l}\text { Transition a } \\
2 F^{\prime}-2 F^{\prime}\end{array}$} & \multicolumn{2}{|c|}{${ }^{79} \mathrm{Br}$} & \multicolumn{2}{|c|}{${ }^{81} \mathrm{Br}$} & \multirow{2}{*}{$\begin{array}{l}\text { Transition a } \\
2 F^{\prime}-2 F^{\prime}\end{array}$} & \multicolumn{2}{|c|}{${ }^{79} \mathrm{Br}$} & \multicolumn{2}{|c|}{${ }^{81} \mathrm{Br}$} \\
\hline & Observed $^{\mathrm{b}}$ & $\Delta v^{c}$ & Observed $^{\mathrm{b}}$ & $\Delta v^{\mathrm{c}}$ & & Observed $^{\mathrm{b}}$ & $\Delta v^{c}$ & Observed $^{\mathrm{b}}$ & $\Delta v^{c}$ \\
\hline $5_{1,5}-4_{1,4}$ & & & & & $81,8-7_{1,7}$ & & & & \\
\hline $13-11$ & 8912.9115 & 0.0059 & 8842.7338 & -0.0008 & $19-17$ & 14264.0051 & 0.0067 & 14151.1551 & 0.0034 \\
\hline $11-9$ & 8917.2336 & -0.0030 & 8846.3813 & -0.0026 & $17-15$ & 14265.0905 & 0.0072 & 14152.0650 & 0.0013 \\
\hline $7-5$ & 8917.9395 & 0.0000 & 8846.9959 & -0.0015 & $13-11$ & 14266.3541 & 0.0017 & 14153.1341 & -0.0003 \\
\hline $9-7$ & 8922.5465 & -0.0032 & 8850.8468 & -0.0003 & $15-13$ & 14267.4790 & 0.0004 & 14154.0781 & 0.0025 \\
\hline $50,5-4_{0,4}$ & & & & & $80,8-7_{0,7}$ & & & & \\
\hline $11-9$ & 8991.0185 & 0.0010 & 8919.5401 & -0.0085 & $17-15$ & 14381.8755 & 0.0016 & 14268.6589 & 0.0016 \\
\hline $13-11$ & 8991.1192 & 0.0022 & 8919.5946 & -0.0058 & $13-11$ & 14383.7798 & 0.0068 & 14270.1521 & -0.0010 \\
\hline $7-5$ & 8999.9237 & -0.0020 & 8926.7245 & -0.0005 & $19-17$ & 14385.2952 & 0.0006 & 14270.6185 & 0.0016 \\
\hline $9-7$ & 9001.9816 & -0.0016 & 8928.2013 & 0.0015 & $15-13$ & 14387.3523 & 0.0007 & 14272.3940 & 0.0002 \\
\hline $5_{1,4}-4_{1,3}$ & & & & & $82,7-7_{2,6}$ & & & & \\
\hline $13-11$ & 9068.7456 & 0.0017 & 8996.1026 & 0.0075 & $19-17$ & & & 14272.9079 & -0.0076 \\
\hline $7-5$ & 9070.5355 & -0.0014 & 8997.7064 & 0.0038 & $13-11$ & 14388.2632 & -0.0045 & 14273.6113 & 0.0005 \\
\hline $11-9$ & 9072.8370 & -0.0001 & 8999.6000 & 0.0081 & $17-15$ & 14391.7346 & -0.0023 & 14276.4978 & 0.0009 \\
\hline $9-7$ & 9075.5248 & -0.0018 & 9001.8158 & 0.0072 & $15-13$ & 14392.6912 & -0.0004 & 14277.3003 & -0.0009 \\
\hline $6_{1,6}-5_{1,5}$ & & & & & $82,6-7_{2,5}$ & & & & \\
\hline $15-13$ & 10696.9826 & 0.0048 & 10612.5414 & -0.0009 & $19-17$ & & & 14276.9245 & 0.0028 \\
\hline $13-11$ & 10699.5003 & 0.0071 & 10614.6598 & 0.0004 & $13-11$ & & & 14277.6365 & -0.0070 \\
\hline $9-7$ & 10700.8613 & -0.0013 & 10615.8164 & -0.0027 & $17-15$ & & & 14280.3469 & -0.0009 \\
\hline $11-9$ & & & 10618.0236 & -0.0008 & $15-13$ & & & 14281.1765 & -0.0018 \\
\hline $60,6-5_{0,5}$ & & & & & $81,7-7_{1,6}$ & & & & \\
\hline $13-11$ & 10789.4393 & 0.0006 & 10703.5841 & -0.0049 & $15-13$ & 14500.2722 & -0.0029 & 14386.8861 & -0.0016 \\
\hline $15-13$ & 10789.6415 & 0.0013 & 10703.6948 & -0.0085 & $19-17$ & 14501.2965 & -0.0007 & 14387.7496 & 0.0029 \\
\hline $11-9$ & 10791.7252 & -0.0017 & 10705.5616 & -0.0010 & $17-15$ & 14512.8220 & -0.0059 & 14396.1940 & 0.0010 \\
\hline $9-7$ & 10791.8906 & -0.0039 & 10705.7356 & -0.0024 & $13-11$ & 14513.4664 & 0.0014 & 14396.6872 & -0.0039 \\
\hline $6_{2,5}-5_{2,4}$ & & & & & $9_{1,9}-8_{1,8}$ & & & & \\
\hline $9-7$ & 10787.5119 & 0.0007 & & & $21-19$ & 16047.1238 & 0.0050 & 15920.1131 & 0.0069 \\
\hline $15-13$ & 10787.7801 & -0.0028 & 10702.3537 & -0.0056 & $19-17$ & 16047.8958 & -0.0018 & 15920.7644 & 0.0038 \\
\hline
\end{tabular}




\begin{tabular}{|c|c|c|c|c|c|c|c|c|c|}
\hline $13-11$ & 10797.9871 & 0.0007 & 10710.9125 & -0.0065 & $15-13$ & 16048.9936 & -0.0088 & 15921.6936 & -0.0002 \\
\hline $11-9$ & 10798.1613 & 0.0282 & 10711.0252 & -0.0020 & $17-15$ & 16049.8097 & 0.0007 & 15922.3698 & 0.0019 \\
\hline $6_{2,4}-5_{2,3}$ & & & & & $9_{0,9-8}-8_{0,8}$ & & & & \\
\hline $9-7$ & & & & & $21-19$ & 16182.4387 & 0.0017 & 16053.4238 & -0.0003 \\
\hline $15-13$ & & & & & $17-15$ & 16184.7320 & -0.0055 & 16055.3081 & -0.0026 \\
\hline $13-11$ & 10799.5924 & -0.0045 & 10712.4962 & 0.0006 & $19-17$ & 16193.5770 & 0.0028 & 16061.4810 & -0.0020 \\
\hline $11-9$ & 10799.7604 & -0.0057 & 10712.6157 & -0.0059 & $15-13$ & 16198.3961 & 0.0029 & 16065.5522 & -0.0003 \\
\hline $6_{1,5}-5_{1,4}$ & & & & & $9_{2,8}-8_{2,7}$ & & & & \\
\hline $15-13$ & 10884.1662 & 0.0001 & 10796.6379 & 0.0082 & $21-19$ & & & 16057.6007 & 0.0002 \\
\hline $9-7$ & 10885.2886 & -0.0031 & 10797.7427 & -0.0001 & $15-13$ & & & 16058.2570 & -0.0046 \\
\hline $13-11$ & 10885.9917 & -0.0027 & 10798.3089 & 0.0069 & $19-17$ & & & 16060.1069 & -0.0009 \\
\hline $11-9$ & 10888.7740 & -0.0009 & 10800.5068 & 0.0003 & $17-15$ & & & 16060.8517 & -0.0008 \\
\hline $7_{1,7}-6_{1,6}$ & & & & & $9_{2,7}-8_{2,6}$ & & & & \\
\hline $17-15$ & 12480.6457 & 0.0046 & 12381.9836 & 0.0004 & 21-19 & & & 16063.2803 & -0.0006 \\
\hline $15-13$ & 12482.2325 & -0.0049 & 12383.3253 & -0.0007 & $15-13$ & & & 16063.9891 & -0.0055 \\
\hline $11-9$ & 12483.6353 & -0.0022 & 12384.5048 & -0.0027 & $19-17$ & & & & \\
\hline $13-11$ & 12485.3003 & -0.0006 & 12385.8992 & 0.0013 & $17-15$ & & & 16066.4093 & -0.0016 \\
\hline $70,7-6_{0,6}$ & & & & & $9_{1,8}-8_{1,7}$ & & & & \\
\hline $15-13$ & 12587.1071 & 0.0011 & 12487.0207 & 0.0037 & $19-17$ & 16327.2156 & -0.0073 & 16195.8566 & -0.0070 \\
\hline $17-15$ & 12587.6942 & 0.0024 & 12487.3716 & 0.0004 & $15-13$ & 16327.2539 & -0.0066 & & \\
\hline $11-9$ & 12589.5177 & -0.0017 & 12489.0808 & 0.0012 & $21-19$ & 16329.9484 & 0.0063 & 16198.2563 & -0.0036 \\
\hline $13-11$ & 12590.3416 & -0.0005 & 12489.6370 & -0.0025 & $17-15$ & 16334.1578 & -0.0036 & 16201.8415 & 0.0026 \\
\hline $72,6-62,5$ & & & & & $10_{1,10-9}-9$ & & & & \\
\hline $17-15$ & 12587.9515 & 0.0027 & 12487.9218 & 0.0078 & 23-21 & 17830.0280 & -0.0090 & 17688.8823 & 0.0072 \\
\hline $11-9$ & 12588.5329 & -0.0018 & 12488.4382 & 0.0048 & 21-19 & 17830.6170 & -0.0054 & 17689.3740 & 0.0072 \\
\hline $15-13$ & 12594.3366 & -0.0015 & 12493.2761 & 0.0021 & $17-15$ & 17831.5682 & 0.0039 & 17690.1670 & 0.0019 \\
\hline $13-11$ & 12595.1634 & -0.0026 & 12493.9678 & 0.0042 & $19-17$ & 17832.1611 & -0.0075 & 17690.6753 & 0.0050 \\
\hline $7_{2,5}-6_{2,4}$ & & & & & $10_{0,10-9}-9_{0,9}$ & & & & \\
\hline $17-15$ & 12590.7244 & -0.0034 & 12490.5995 & -0.0025 & 17-15 & 17974.3248 & 0.0062 & 17831.6180 & -0.0004 \\
\hline $11-9$ & 12591.3318 & -0.0074 & 12491.1389 & -0.0024 & 21-19 & 17975.6474 & 0.0083 & 17832.7421 & 0.0001 \\
\hline $15-13$ & 12596.9552 & 0.0055 & 12495.8325 & 0.0073 & $23-21$ & 17979.0837 & -0.0072 & 17835.7582 & -0.0049 \\
\hline $13-11$ & 12597.8098 & 0.0061 & 12496.5421 & 0.0071 & $19-17$ & 17980.4312 & -0.0077 & 17836.8700 & -0.0233 \\
\hline $7_{1,6}-6_{1,5}$ & & & & & $10_{1,9}-9_{1,8}$ & & & & \\
\hline $11-9$ & 12699.4975 & -0.0043 & 12597.3206 & -0.0041 & 21-19 & 18140.7439 & 0.0304 & 17994.8142 & 0.0143 \\
\hline $15-13$ & 12699.7167 & -0.0026 & 12597.4573 & 0.0028 & $23-21$ & & & 17994.8622 & 0.0137 \\
\hline $17-15$ & 12701.5726 & -0.0030 & 12598.1316 & 0.0016 & $17-15$ & & & & \\
\hline
\end{tabular}


13-1

12704.7627

$-0.0034$

12600.9360

0.0004

19-17

18142.5385

a Transitions with quantum numbers $\left(J^{\prime} K_{p}^{\prime} K_{o}^{\prime}-J K_{p}^{\prime \prime} K^{\prime \prime}{ }_{o}\right)$ with hyperfine components.

${ }^{b}$ Observed frequency in $\mathrm{MHz}$.

c $\Delta v=v_{\text {obs }}-v_{\text {calc }}$ in $\mathrm{MHz}$ 
Table 5

Observed $a$-type transition frequencies for ga conformer of 1-bromobutane.

\begin{tabular}{|c|c|c|c|c|c|c|c|c|c|}
\hline \multirow{2}{*}{$\begin{array}{l}\text { Transition a } \\
2 F^{\prime}-2 F^{\prime \prime}\end{array}$} & \multicolumn{2}{|c|}{${ }^{79} \mathrm{Br}$} & \multicolumn{2}{|c|}{${ }^{81} \mathrm{Br}$} & \multirow{2}{*}{$\begin{array}{l}\text { Transition a } \\
2 F^{\prime}-2 F^{\prime \prime}\end{array}$} & \multicolumn{2}{|c|}{${ }^{79} \mathrm{Br}$} & \multicolumn{2}{|c|}{${ }^{81} \mathrm{Br}$} \\
\hline & Observed $^{\mathrm{b}}$ & $\Delta v^{c}$ & Observed $^{b}$ & $\Delta v^{c}$ & & Observed $^{b}$ & $\Delta v^{\mathrm{c}}$ & Observed $^{\mathrm{b}}$ & $\Delta v^{\mathrm{c}}$ \\
\hline $4_{1,4-3} 3_{1,3}$ & & & & & $6_{0,6}-5_{0,5}$ & & & & \\
\hline $11-9$ & 9055.6548 & 0.0006 & 8989.1287 & 0.0014 & $9-7$ & 13850.9884 & -0.0035 & 13748.0272 & -0.0019 \\
\hline $9-7$ & 9058.1801 & 0.0044 & 8991.2502 & -0.0048 & $15-13$ & 13851.2811 & 0.0001 & & \\
\hline $5-3$ & 9062.6215 & -0.0044 & 8994.9764 & -0.0083 & $13-11$ & 13852.9299 & -0.0019 & 13749.5532 & 0.0024 \\
\hline $7-5$ & 9064.9112 & 0.0002 & 8996.9363 & -0.0008 & $11-9$ & 13853.8919 & -0.0011 & 13750.3480 & 0.0042 \\
\hline $4_{2,3}-3_{2,2}$ & & & & & $62,5-5_{2,4}$ & & & & \\
\hline $5-3$ & & & 9184.0724 & -0.0037 & $9-7$ & 13883.3884 & 0.0001 & 13779.5112 & 0.0027 \\
\hline $11-9$ & 9258.8655 & -0.0005 & 9190.1306 & 0.0062 & $15-13$ & 13883.7936 & 0.0018 & 13779.7895 & -0.0065 \\
\hline $7-5$ & 9261.6461 & 0.0082 & 9191.7431 & -0.0081 & $13-11$ & 13886.3989 & 0.0027 & & \\
\hline $9-7$ & 9266.2108 & -0.0007 & 9196.0507 & -0.0005 & $11-9$ & 13886.4337 & 0.0022 & & \\
\hline $4_{2,2-32,1}$ & & & & & $62,4-5_{2,3}$ & & & & \\
\hline $5-3$ & 9264.0017 & 0.0097 & 9195.1144 & -0.0042 & $9-7$ & & & 13816.4754 & -0.0070 \\
\hline $11-9$ & 9265.3418 & -0.0019 & 9196.5981 & 0.0053 & $15-13$ & & & 13816.8231 & 0.0015 \\
\hline $7-5$ & 9271.6650 & 0.0004 & 9201.6075 & -0.0006 & $13-11$ & & & 13817.0231 & -0.0042 \\
\hline $9-7$ & 9273.2314 & -0.0075 & 9203.1945 & 0.0010 & $11-9$ & & & 13817.4712 & -0.0010 \\
\hline $4_{1,3-}-3_{1,2}$ & & & & & $6_{1,5}-5_{1,4}$ & & & & \\
\hline $5-3$ & 9451.3218 & -0.0004 & 9380.3553 & 0.0025 & $9-7$ & 14175.6895 & -0.0023 & & \\
\hline $7-5$ & 9456.8867 & -0.0014 & 9384.7055 & -0.0018 & $11-9$ & 14176.3396 & 0.0039 & 14068.2916 & 0.0061 \\
\hline $11-9$ & 9458.5150 & -0.0010 & 9386.1029 & -0.0005 & $15-13$ & 14179.7689 & -0.0012 & 14071.9219 & -0.0005 \\
\hline $9-7$ & 9459.7170 & 0.0001 & 9387.2837 & 0.0071 & $13-11$ & 14180.4958 & 0.0008 & 14072.5946 & 0.0008 \\
\hline $5_{1,5}-4_{1,4}$ & & & & & $7_{1,7}-6_{1,6}$ & & & & \\
\hline $13-11$ & 11319.1409 & 0.0034 & 11235.7973 & 0.0056 & $17-15$ & 15841.0915 & -0.0018 & 15724.3533 & 0.0111 \\
\hline $11-9$ & 11320.6016 & 0.0021 & 11237.0326 & 0.0014 & $15-13$ & 15841.9431 & 0.0056 & 15725.0701 & 0.0010 \\
\hline $7-5$ & 11322.7591 & -0.0026 & 11239.0001 & -0.0024 & $11-9$ & 15842.9293 & 0.0007 & 15725.9180 & -0.0092 \\
\hline $9-7$ & 11323.7861 & -0.0016 & 11239.9892 & 0.0038 & $13-11$ & 15843.9808 & 0.0045 & 15726.7863 & 0.0026 \\
\hline $5_{0,5}-4_{0,4}$ & & & & & $70,7-6_{0,6}$ & & & & \\
\hline $7-5$ & 11550.4714 & -0.0057 & 11464.9397 & -0.0053 & $17-15$ & 16144.0332 & 0.0002 & 16024.0561 & -0.0031 \\
\hline $13-11$ & 11552.2756 & -0.0006 & 11466.1096 & 0.0061 & $11-9$ & & & 16024.2136 & 0.0031 \\
\hline $11-9$ & 11552.4731 & -0.0015 & 11466.4206 & 0.0011 & $15-13$ & 16146.2831 & 0.0033 & 16025.9452 & 0.0012 \\
\hline $9-7$ & 11554.5561 & -0.0002 & 11468.0926 & 0.0006 & $13-11$ & 16147.1338 & 0.0022 & 16026.6282 & 0.0055 \\
\hline $5_{2,4-4} 4_{2,3}$ & & & & & $72,6-62,5$ & & & & \\
\hline $7-5$ & 11569.6564 & -0.0012 & 11483.2814 & -0.0032 & $11-9$ & 16195.2153 & 0.0003 & 16073.9886 & -0.0010 \\
\hline $13-11$ & 11570.6959 & 0.0003 & 11484.1126 & -0.0008 & $17-15$ & 16195.6382 & -0.0087 & 16074.2501 & 0.0036 \\
\hline $9-7$ & 11574.4422 & 0.0009 & 11487.3058 & 0.0002 & $15-13$ & 16197.2177 & -0.0008 & 16075.6493 & 0.0024 \\
\hline
\end{tabular}




\begin{tabular}{|c|c|c|c|c|c|c|c|c|c|}
\hline 11-9 & 11575.0970 & 0.0018 & 11487.8671 & -0.0010 & 13-11 & 16197.6368 & 0.0032 & 16075.9361 & 0.0022 \\
\hline $52,3-4_{2,2}$ & & & & & $72,5-62,4$ & & & & \\
\hline $7-5$ & 11591.8197 & -0.0033 & 11504.7440 & -0.0045 & $15-13$ & & & 16131.9214 & 0.0044 \\
\hline $13-11$ & 11592.6139 & -0.0010 & 11505.3668 & -0.0030 & $11-9$ & & & 16132.4952 & 0.0033 \\
\hline $9-7$ & 11594.8935 & 0.0067 & 11507.3418 & 0.0078 & $17-15$ & & & 16134.0856 & 0.0033 \\
\hline $11-9$ & 11595.1040 & 0.0003 & 11507.5630 & 0.0034 & $13-11$ & & & 16134.3334 & 0.0062 \\
\hline $5_{1,4}-4_{1,3}$ & & & & & $7_{1,6}-6_{1,5}$ & & & & \\
\hline $7-5$ & 11817.9631 & -0.0079 & 11728.6740 & -0.0023 & $11-9$ & 16536.3008 & -0.0039 & 16411.1625 & -0.0067 \\
\hline $13-11$ & 11819.4552 & -0.0026 & 11729.4188 & 0.0026 & $13-11$ & 16538.2071 & -0.0030 & 16412.6120 & -0.0083 \\
\hline $11-9$ & 11820.4209 & 0.0028 & 11730.3054 & 0.0048 & $17-15$ & 16538.4708 & -0.0027 & 16412.8188 & -0.0024 \\
\hline $9-7$ & 11822.0931 & -0.0057 & 11732.3155 & -0.0037 & $15-13$ & 16538.8783 & -0.0007 & 16413.2615 & -0.0028 \\
\hline $6_{1,6}-5_{1,5}$ & & & & & $8_{1,8}-7_{1,7}$ & & & & \\
\hline $15-13$ & 13580.9637 & 0.0026 & 13480.8851 & 0.0036 & $19-17$ & 18099.4241 & 0.0033 & 17966.0580 & 0.0064 \\
\hline $13-11$ & 13582.0098 & 0.0021 & 13481.7753 & 0.0024 & $17-15$ & 18100.0999 & -0.0024 & 17966.6586 & -0.0012 \\
\hline $9-7$ & 13585.0390 & -0.0020 & 13484.1128 & -0.0001 & $13-11$ & 18100.7189 & -0.0062 & 17967.1954 & -0.0086 \\
\hline $11-9$ & 13586.9736 & 0.0030 & 13485.5731 & 0.0048 & $15-13$ & 18101.9086 & -0.0003 & 17968.1290 & -0.0169 \\
\hline
\end{tabular}

a Transitions with quantum numbers $\left(J^{\prime} K_{p}^{\prime} K_{o}^{\prime}-J K_{p}^{\prime \prime} K^{\prime \prime}{ }_{o}\right)$ with hyperfine components.

${ }^{\mathrm{b}}$ Observed frequency in $\mathrm{MHz}$

${ }^{c} \Delta v=v_{\text {obs }}-v_{\text {calc }}$ in MHz. 
Table 6

Observed $b$-type transition frequencies for ga conformer of 1-bromobutane.

\begin{tabular}{|c|c|c|c|c|}
\hline \multirow{2}{*}{$\begin{array}{c}\text { Transition a } \\
\text { 2F'-2F"' }\end{array}$} & \multicolumn{2}{|c|}{${ }^{79} \mathrm{Br}$} & \multicolumn{2}{|c|}{${ }^{81} \mathrm{Br}$} \\
\hline & Observed $^{\mathrm{b}}$ & $\Delta v^{c}$ & Observed $^{\mathrm{b}}$ & $\Delta v^{c}$ \\
\hline \multicolumn{5}{|l|}{$5_{1,4-5_{0,5}}$} \\
\hline $11-11$ & 7777.7438 & -0.0035 & 7770.1798 & -0.0098 \\
\hline $9-9$ & 7797.3636 & -0.0060 & 7787.3211 & 0.0067 \\
\hline $13-13$ & 7837.3200 & 0.0016 & 7819.7357 & 0.0079 \\
\hline 7-7 & 7854.1477 & 0.0036 & 7834.3673 & -0.0005 \\
\hline \multicolumn{5}{|l|}{$6_{1,5}-6_{0,6}$} \\
\hline $13-13$ & 8105.3067 & -0.0037 & 8093.2313 & -0.0012 \\
\hline $11-11$ & 8119.8144 & 0.0020 & 8105.2659 & 0.0099 \\
\hline $15-15$ & 8165.8077 & 0.0002 & 8143.5195 & 0.0015 \\
\hline 9-9 & 8178.8477 & 0.0037 & 8154.5293 & -0.0008 \\
\hline \multicolumn{5}{|l|}{$7_{1,6}-7_{0,7}$} \\
\hline $15-15$ & 8497.9049 & -0.0048 & 8480.5485 & -0.0043 \\
\hline $13-13$ & 8510.8930 & 0.0020 & 8491.2583 & 0.0047 \\
\hline $17-17$ & 8560.2492 & 0.0011 & 8532.2748 & -0.0051 \\
\hline $11-11$ & 8571.0043 & -0.0003 & 8541.4853 & -0.0035 \\
\hline \multicolumn{5}{|l|}{$81,7-8_{0,8}$} \\
\hline $17-17$ & 8958.5182 & 0.0015 & 8935.9656 & 0.0040 \\
\hline $15-15$ & 8973.3489 & 0.0037 & 8947.4657 & -0.0059 \\
\hline $19-19$ & 9025.7020 & 0.0019 & & \\
\hline 13-13 & 9031.4115 & -0.0038 & & \\
\hline \multicolumn{5}{|l|}{$1_{1,1-1} 0_{0,0}$} \\
\hline $5-3$ & 9295.2984 & 0.0089 & & \\
\hline 3-3 & 9328.5355 & 0.0000 & & \\
\hline \multicolumn{5}{|l|}{$7_{0,7}-6_{1,6}$} \\
\hline $15-13$ & 10068.7911 & 0.0072 & 9944.8328 & 0.0048 \\
\hline $13-11$ & 10078.4091 & 0.0016 & 9953.0471 & -0.0091 \\
\hline $17-15$ & 10110.2837 & -0.0064 & 9979.6186 & 0.0006 \\
\hline $11-9$ & & & 9989.6906 & -0.0068 \\
\hline \multicolumn{5}{|l|}{$8_{0,8}-7_{1,7}$} \\
\hline $17-15$ & 12659.1835 & 0.0021 & & \\
\hline $15-13$ & 12667.0689 & 0.0076 & & \\
\hline $19-17$ & 12698.8604 & -0.0066 & & \\
\hline $13-11$ & 12709.1499 & 0.0017 & & \\
\hline \multicolumn{5}{|l|}{$9_{0,9-8}-8_{1,8}$} \\
\hline 19-17 & & & 15105.1720 & -0.0084 \\
\hline $17-15$ & & & 15110.6603 & 0.0012 \\
\hline $21-19$ & & & 15136.6024 & 0.0093 \\
\hline $15-13$ & & & 15143.8402 & 0.0061 \\
\hline \multicolumn{5}{|l|}{$5_{1,5}-4_{0,4}$} \\
\hline $7-5$ & & & 17763.3748 & 0.0007 \\
\hline $13-11$ & & & 17777.7998 & 0.0045 \\
\hline $9-7$ & & & 17806.4318 & -0.0022 \\
\hline \multicolumn{5}{|l|}{$31,3-20,2$} \\
\hline \multicolumn{5}{|l|}{ 3-1 } \\
\hline $9-7$ & 13656.8066 & -0.0025 & 13608.7323 & -0.0083 \\
\hline $5-3$ & 13668.7169 & 0.0011 & 13619.1693 & 0.0081 \\
\hline $7-5$ & 13703.1242 & 0.0004 & 13647.3021 & 0.0001 \\
\hline
\end{tabular}

a Transitions with quantum numbers $\left(J^{\prime} K_{p}^{\prime} K_{o}^{\prime}-J K_{p}^{\prime \prime} K^{\prime \prime}{ }_{o}\right)$ with hyperfine components.

${ }^{\mathrm{b}}$ Observed frequency in MHz.

${ }^{\mathrm{c}} \Delta v=v_{\mathrm{obs}}-v_{\text {calc }}$ in MHz. 
Table 7

Observed $a$-type transition frequencies for $\mathbf{g g}$ conformer of 1-bromobutane.

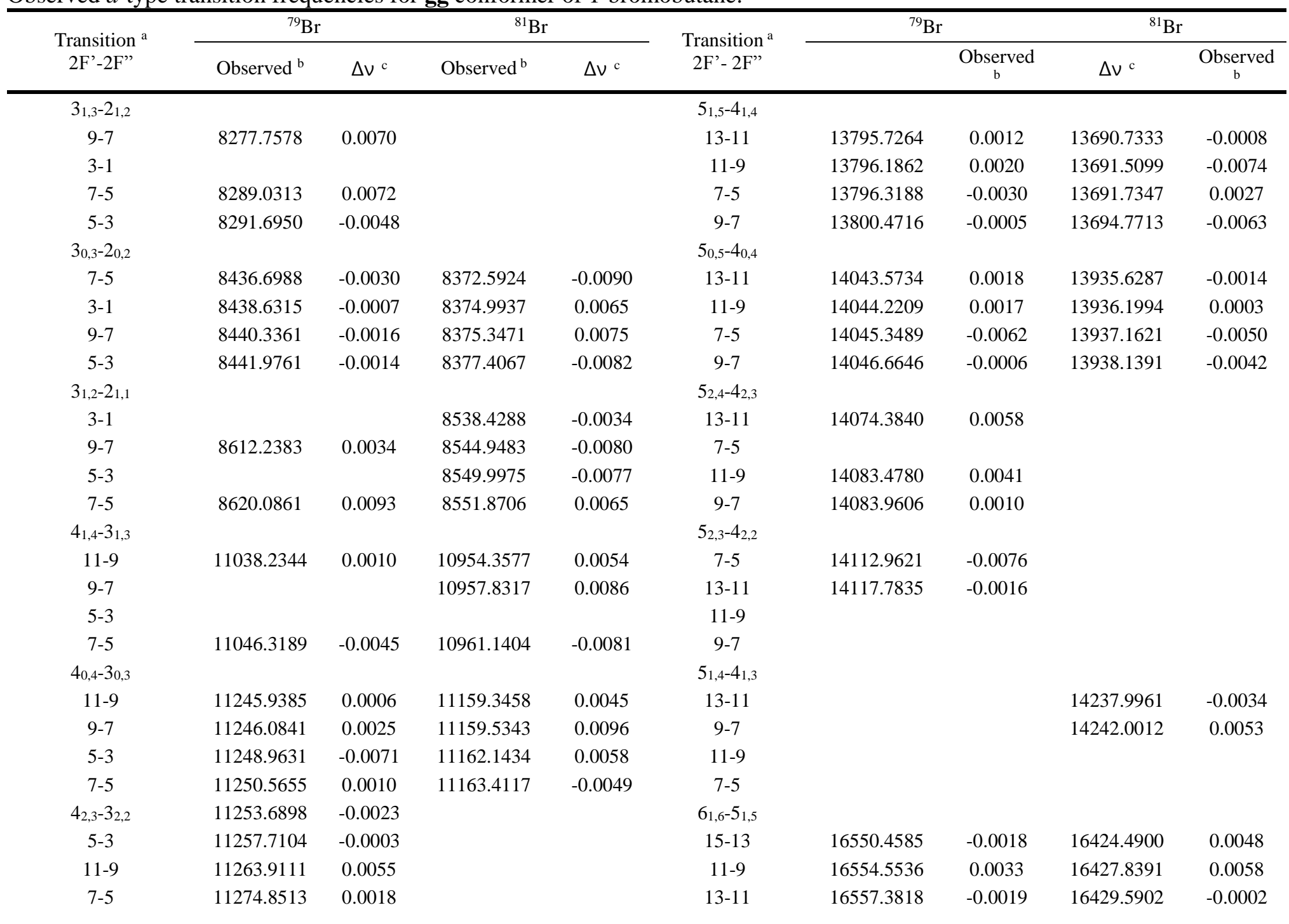




\begin{tabular}{|c|c|c|c|c|c|c|c|c|c|}
\hline $9-7$ & & & & & $9-7$ & 16560.4792 & -0.0014 & 16431.9293 & -0.0041 \\
\hline $4_{2,2-2}-3_{2,1}$ & & & & & $60,6-50,5$ & & & & \\
\hline $5-3$ & 11273.2148 & -0.0013 & & & $15-13$ & 16831.6794 & 0.0044 & & \\
\hline $11-9$ & 11272.8792 & -0.0002 & & & $13-11$ & 16832.5083 & 0.0038 & & \\
\hline $7-5$ & & & & & $9-7$ & 16832.7757 & -0.0043 & & \\
\hline $9-7$ & 11292.7704 & -0.0070 & & & $11-9$ & 16834.3459 & 0.0012 & & \\
\hline $4_{1,3-}-3_{1,2}$ & & & & & $66_{1,5}-5_{1,4}$ & & & & \\
\hline $5-3$ & 11480.5309 & -0.0074 & 11391.3173 & -0.0014 & $9-7$ & & & & \\
\hline $11-9$ & 11482.7882 & 0.0028 & 11392.8452 & 0.0013 & $13-11$ & & & & \\
\hline $9-7$ & 11485.5067 & 0.0015 & 11395.3620 & 0.0058 & $15-13$ & & & 17080.1688 & -0.0066 \\
\hline $7-5$ & 11487.0037 & -0.0008 & 11396.4026 & 0.0034 & $11-9$ & & & 17080.3418 & 0.0051 \\
\hline
\end{tabular}

a Transitions with quantum numbers $\left(J^{\prime} K_{p}^{\prime} K_{o}^{\prime}-J K_{p}^{\prime \prime} K^{\prime \prime}{ }_{o}\right)$ with hyperfine components.

${ }^{\mathrm{b}}$ Observed frequency in $\mathrm{MHz}$.

${ }^{c} \Delta v=v_{\text {obs }}-v_{\text {calc }}$ in $\mathrm{MHz}$ 
Table 8

Comparisons of $\mathrm{Br}$ quadrupole coupling tensors, in the principal axes for four alkyl bromide ( $\mathrm{R}-\mathrm{Br})$ including 1-bromobutane.

\begin{tabular}{|c|c|c|c|c|c|c|c|c|}
\hline \multirow[b]{2}{*}{ Molecule } & \multirow[b]{2}{*}{ Molecular formula } & \multicolumn{3}{|c|}{${ }^{79} \mathrm{Br}$} & \multicolumn{3}{|c|}{${ }^{81} \mathrm{Br}$} & \multirow[b]{2}{*}{ Ref. } \\
\hline & & $\chi_{x x}$ & $\chi_{\mathrm{yy}}$ & $\chi_{z z}$ & $\chi_{x x}$ & $\chi_{\mathrm{yy}}$ & $\chi_{\mathrm{zz}}$ & \\
\hline bromomethane & $\mathrm{CH}_{3} \mathrm{Br}$ & $-288.5544^{a}$ & $-288.5544^{a}$ & $577.1088(57)$ & $-241.0515^{a}$ & $-241.0515^{a}$ & $482.1030(94)$ & {$[15,16]$} \\
\hline \multirow[t]{2}{*}{ bromoethane } & $\mathrm{CH}_{3} \mathrm{CH}_{2} \mathrm{Br}$ & $-270.32(166)$ & $-273.71(17)$ & $544.03(168)$ & $-225.25(201)$ & $-228.65(18)$ & 453.91(203) & [12] \\
\hline & & -266.91 & -273.55 & 540.46 & -221.09 & -228.60 & 449.69 & [13] \\
\hline 1-bromopropane & $\mathrm{CH}_{3} \mathrm{CH}_{2} \mathrm{CH}_{2} \mathrm{Br}$ (gauche) & -279.0 & -247.4 & 526.4 & -228.1 & -206.0 & 434.1 & [14] \\
\hline \multirow[t]{3}{*}{ 1-bromobutane } & $\mathrm{CH}_{3} \mathrm{CH}_{2} \mathrm{CH}_{2} \mathrm{CH}_{2} \mathrm{Br}(a a)$ & $-269.48(48)$ & $-273.97(59)$ & $543.45(32)$ & $-225.51(48)$ & $-228.44(58)$ & $453.95(33)$ & This work \\
\hline & $(g a)$ & $-264.89(197)$ & $-274.83(154)$ & $539.71(63)$ & $-220.16(242)$ & $-230.95(210)$ & $451.11(84)$ & This work \\
\hline & $(g g)$ & $-264.88(54)$ & $-271.29(39)$ & $536.17(46)$ & $-221.98(182)$ & $-226.31(393)$ & $448.29(465)$ & This work \\
\hline
\end{tabular}

a by symmetry

Table 9

The measured and calculated dipole moment for aa conformer of $\mathrm{CH}_{3} \mathrm{CH}_{2} \mathrm{CH}_{2} \mathrm{CH}_{2}{ }^{79} \mathrm{Br}$.

\begin{tabular}{|c|c|c|c|}
\hline & aa $a_{\text {obs }}$ & aacalc & $\Delta_{\text {obs-calc }}{ }^{c}$ \\
\hline$\mu_{\mathrm{a}}(\mathrm{D})$ & $2.229(13)$ & 2.179 & 0.050 \\
\hline$\mu_{\mathrm{b}}(\mathrm{D})$ & $0.603(60)$ & 0.744 & -0.141 \\
\hline$\mu_{\mathrm{c}}(\mathrm{D})^{\mathrm{a}}$ & 0.000 & 0.000 & 0.000 \\
\hline$\mu_{\mathrm{tot}}(\mathrm{D})$ & 2.309 & 2.303 & 0.006 \\
\hline
\end{tabular}

$\overline{{ }^{a}} \mu_{c}$ is not fitted because the aa conformer has an $a b$ plane of symmetry, $\mu_{\mathrm{c}}$ dipole component is zero due to symmetry

${ }^{\mathrm{b}}$ The number of distinct Stark measurements.

${ }^{\mathrm{c}} \Delta_{\text {obs-calc }}$ is observed minus calculated dipole moment value in $\mathrm{D}$. 

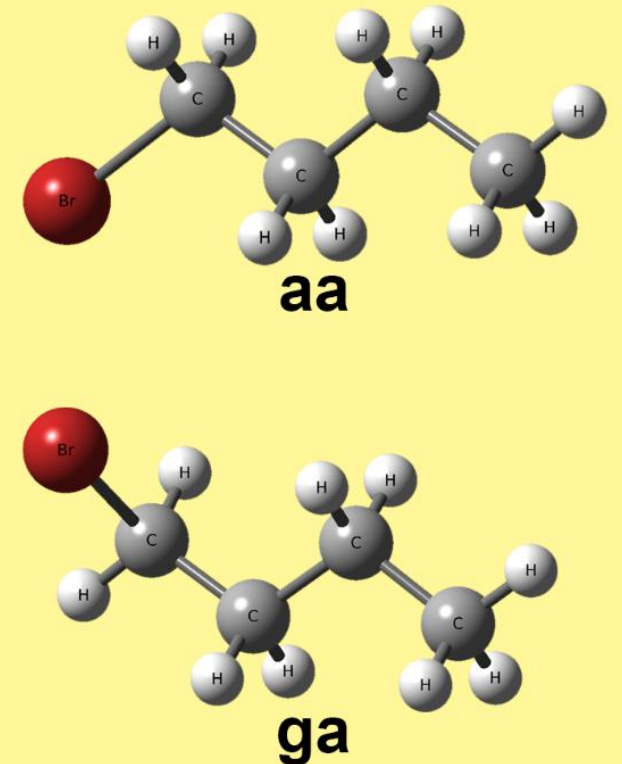

Five structural isotopomers for 1-bromobutane

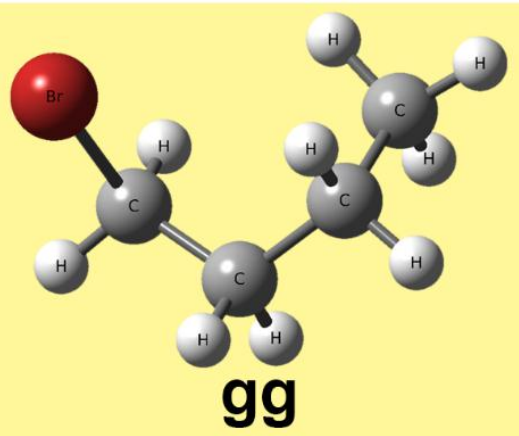

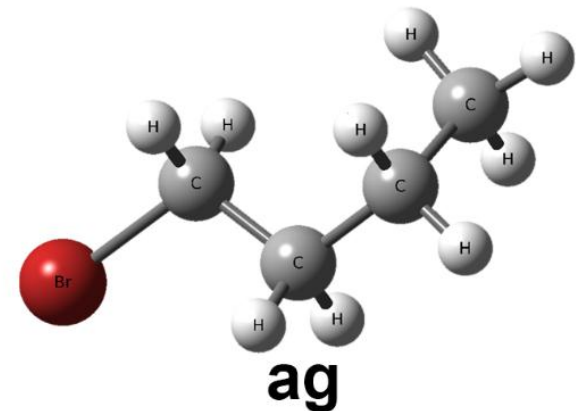

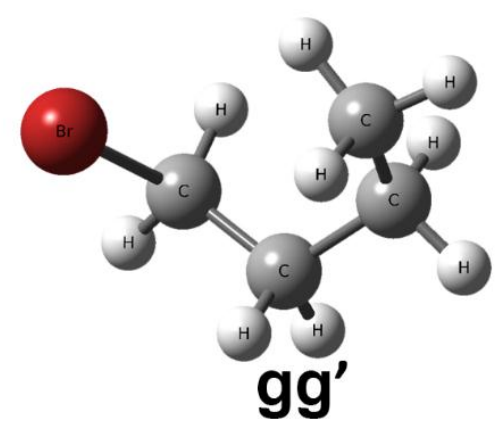

